\title{
Desenvolvimento de uma aplicação web para auxiliar no ensino da Matemática para alunos do Ensino Fundamental
}

\author{
Armando M. Toda ${ }^{1}$, Roberto S. do Carmo ${ }^{1}$, \\ João Coelho Neto ${ }^{2}$, Ana Lucia Silva ${ }^{1}$, Jacques D. Brancher ${ }^{1}$ \\ ${ }^{1}$ Universidade Estadual de Londrina (UEL) \\ Caixa Postal 86057-970 \\ Londrina - PR - Brazil \\ ${ }^{2}$ Universidade Estadual do Norte do Paraná Campus de Cornélio Procópio \\ Cornélio Procópio - PR - Brazil \\ \{armando.toda, robertodocarmo\}@gmail.com \\ joaocoelho@uenp.edu.br, \{analucia, jacques\}@uel.br
}

\begin{abstract}
This paper presents the development of a web application focused for training math concepts in elementary school students. The system allows students solve a series of problems divided according to the classification used by OBMEP, as themes, sub-themes and levels. The application is implemented with Gamification concepts. The problems were selected using a method developed by the authors, described in the article, and had their variables generalized to ensure that the student does not memorize the answer, but the logic for resolving the issue, i.e, the identification of the mathematical algorithm proposed.
\end{abstract}

Resumo. Este trabalho visa apresentar o desenvolvimento de uma aplicação web direcionada para o treinamento dos conceitos de matemática em alunos do Ensino Fundamental. O sistema permite aos discentes resolver uma série de problemas divididos de acordo com a classificação utilizada pela $O B M E P^{1}$, em temas, sub-temas e níveis. A aplicação está implementada com conceitos de Gamification. Os problemas foram selecionados utilizando um método criado pelos autores, descrito no corpo do artigo, e tiveram suas variáveis generalizadas para garantir que o discente não memorize a resposta, e sim a lógica para a resolução da questão, ou seja, na identificação do algoritmo matemático proposto.

\section{Introdução}

O Ensino Básico no país vem enfrentando dificuldades nos últimos anos, principalmente na matemática uma vez que estudantes brasileiros obtiveram o $58^{\circ}$ lugar na classificação do Programme for International Student Assessment (PISA). Este resultado foi justificado pela falta de habilidades cognitivas e interpretativas dos estudantes, no Ensino Básico, de aplicarem os conceitos aprendidos em sala de aula na prática [PISA,2012].

Uma das tentativas para modificar este resultado foi a criação da OBMEP. Esta iniciativa é promovida pelo Ministério da Educação (MEC) e Ministério de Ciência e Tecnologia (MCT), realizada pelo Instituto de Matemática Pura e Aplicada (IMPA) em parceria com a Sociedade Brasileira de Matemática (SBM). Desde o início das Olimpíadas,

\footnotetext{
${ }^{1}$ Olimpíada Brasileira de Matemática das Escolas Públicas
} 
vários estudantes foram beneficiados com recursos para continuarem os estudos na área. O evento foca no melhoramento do estudo da matemática nas escolas públicas e a qualidade da Educação Básica [OBMEP, s.a.].

Nos últimos anos houve um aumento na emergência de tecnologias digitais da informação e comunicação (TDIC) direcionadas à auxiliar nos processos educacionais. Pesquisas recentes revelam que a utilização destas proporcionou o surgimento de novas técnicas de ensino e um aumento considerável na performance dos alunos, especialmente quando ligadas a conceitos presentes no cotidiano dos discentes [Generoso et al., 2013] [Pietruchinski et al., 2011].

Um exemplo destas técnicas é a Gamification. Este conceito pode ser definido como a utilização de conceitos de jogos fora do seu escopo principal [McGonigal, 2011] [Zichermann and Cunningham, 2011]. Quando utilizada corretamente, esta pode provocar mudanças positivas no cenário educacional, promovendo uma maior interação e engajamento dos discentes [Lee and Hammer, 2011].

Considerado o que foi exposto, este trabalho visa demonstrar o projeto e o desenvolvimento de um sistema web para auxiliar no ensino da matemática. Inicialmente abrangendo o conteúdo de aritmética do Ensino Fundamental, voltado à discentes do quinto e sexto ano.

Este trabalho está dividido em: Seção 2 apresenta os conceitos relacionados a Gamification e sua aplicação na educação. Seção 3 descreve o método de projeto enquanto a seção 4 demonstra o desenvolvimento da aplicação, assim como a proposta de estudo. A seção 5 apresenta uma breve discussão e por fim, a seção 6 apresenta as conclusões e trabalhos futuros.

\section{Gamification}

O termo, além do conceito exposto anteriormente, pode ser considerado um processo motivacional que estimula o engajamento do indivíduo, podendo ser aplicada em diferentes áreas como educação, saúde e economia [Zichermann and Cunningham, 2011] [Lee and Hammer, 2011].

Este método surgiu a partir dos estudos realizados para identificar as propriedades engajadoras que os jogos provocavam na grande maioria dos seus usuários. Alguns autores atestam que os primórdios dessa técnica surgiram no período da guerra fria.

Segundo [Nelson, 2012], a corrida socialista estimulada pela União Soviética (URSS) utilizava meios não materiais para bonificar seus trabalhadores pelo esforço desempenhado. Dentre estes meios encontravam-se medalhas de mérito. Isto se deve ao fato de que os princípios defendidos pelo país não admitiam o uso de capital para quantificar o trabalho do indivíduo.

Após os eventos da Guerra Fria, pesquisadores norte-americanos elaboraram teorias sobre estes sistemas de bonificação utilizado pela URSS. Os estudos tinham o intuito de criar novas metodologias para aumentar a produção e diminuir os custos dos empregados, por meio do que inicialmente chamaram de "Funfication" [Nelson, 2012] .

Em função disto, vários estudos foram realizados para relacionar jogos e produtividade e, em meados de 2008, o termo Gamification surgiu na mídia 
[Deterding et al., 2011]. Porém, antes de adentrar nos conceitos apresentados pelo método, é necessário primeiramente definir o jogo digital, ou game.

De acordo com [Salen and Zimmerman, 2003], um jogo pode ser definido como um sistema que engaja o jogador em um conflito virtual, seguindo um conjunto de regras específicas e com um resultado quantificavelmente pré-determinado. Já para [Kapp, 2012], é um sistema que é utilizado por conta do feedback instantâneo e interação constante que provoca uma reação emocional no jogador, relacionados ao conceito anterior.

Alguns autores debatem estes impactos emocionais e psicológicos ocasionados por esses jogos e visualizados quando aplicamos estes conceitos em outras atividades. [Csikszentmihalyi, 2008] propôs a teoria do fluxo (Flow Theory) que consiste em engajar o indivíduo por meio de suas habilidades e a dificuldade das atividades realizadas.

Quando ambos estão em balanço, o indivíduo consegue entrar em um estado de fluidez que permite-o realizar uma determinada tarefa. Em jogos, este conceito é bem visível, pois a medida que o jogador progride suas habilidades, também ocorre o mesmo com a dificuldade do jogo.

Este é visualizável no sistema uma vez que as o nível das atividades progride de acordo com as habilidades do aluno. Este é o responsável por determinar a dificuldade das questões baseando-se no seu conhecimento acerca do assunto. Espera-se que o usuário entre no estado descrito pela teoria de [Csikszentmihalyi, 2008].

Após a definição inicial, pode-se extrair os mecânicas previamente citados e que podem ser utilizados para o engajamento do indivíduo.[Dignan, 2011] extraiu alguns destes e os dividiu em diversas áreas. Estas são utilizadas para determinar os impactos provocados nos usuários com ou sem a sua utilização (Tabela 1).

Tabela 1. Conceitos de jogos extraídos por [Dignan, 2011]

\begin{tabular}{|l|l|l|l|}
\hline Objetivos & Competição & Oportunidades & Classificação \\
\hline Pressão Temporal & Escassez & Puzzles & Reconhecimento \\
\hline Novidades & Níveis & Pressão Social & Sensação \\
\hline Cooperação & Moeda de troca & Renovação & Pontos \\
\hline Julgamento & Dados & Progresso & \\
\hline
\end{tabular}

Conforme pode ser observado na tabela 1 apresenta as mecânicas encontradas por [Dignan, 2011]. Segundo autores como [Zichermann and Cunningham, 2011], é necessário realizar um estudo sobre o público alvo para selecionar os conceitos a serem aplicados, para que a Gamification provoque o efeito positivo que deveria. O sistema utilizará alguns destes com o intuito de engajar e motivar o aluno a realizar as atividades contidas na base de dados.

\section{Método}

O sistema consiste em uma aplicação web modelada como plataforma social, onde estudantes tem acesso a diversos problemas matemáticos. Estes problemas são classificados de acordo com a divisão realizada nas OBMEP, em relação aos temas e níveis. Os subtemas dos problemas foram classificados em conjunto com os professores de matemática que auxiliaram no desenvolvimento do sistema. 
Os problemas do sistema foram extraídos das bases de questões utilizadas pelas olimpíadas. Cada questão foi analisada a ponto de ter sua fórmula generalizada, a ponto de gerar resultados diferentes a cada execução (Figura 1). Isto, na prática, significa que o discente poderá realizar a mesma questão que outro, no entanto os valores são diferentes, alterando o resultado final.

Isto foi realizado com o intuito de garantir o conceito da novidade, uma vez que os problemas terão resultados diferentes, porém utilizam a mesma lógica na resolução. A partir disto, espera-se que os discentes adquiram o raciocínio ao invés de apenas "decorar" a solução.

As questões utilizadas foram extraídas baseadas em um método criado pelos autores para identificá-las e classificá-las de acordo com a divisão citada anteriormente. Este processo foi acompanhado por docentes da área e pode ser observado na figura 1. Inicialmente foi escolhida a área da Aritmética, direcionada ao Ensino Fundamental I, para a seleção e classificação dos sub-temas dos problemas.

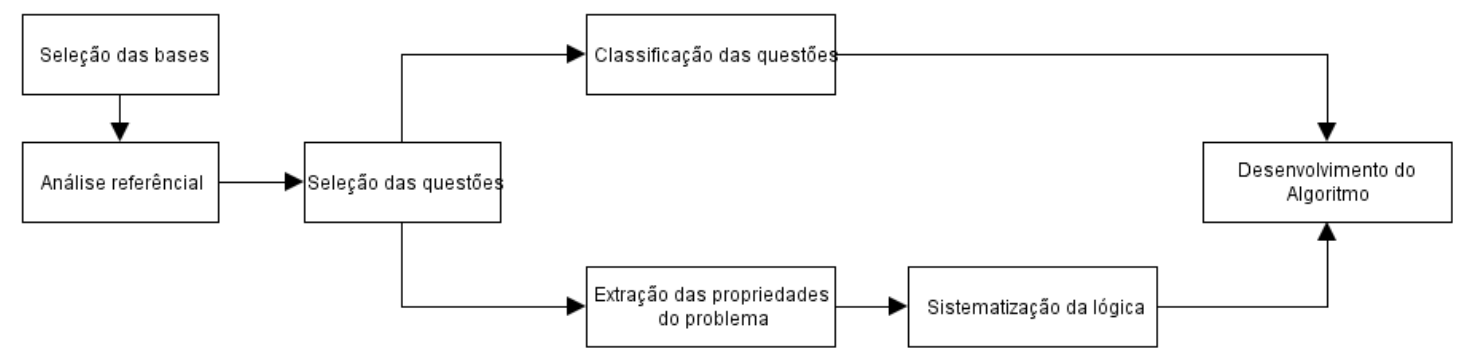

Figura 1. Método de seleção dos problemas

O primeiro estágio consiste na seleção do banco de questões à ser analisado. Inicialmente foi escolhido o banco da OBMEP de 2010, por ser o mais robustos em número de questões . Em seguida foi realizada uma análise referencial, com o intuito de averiguar os sub-temas encontrados em cada um dos bancos (Tabela 2).

Tabela 2. Classificação dos sub-temas da área de Aritmética

\begin{tabular}{|l|l|}
\hline Operações Básicas & Múltiplos e Divisores \\
\hline Fatoração & Mínimo Divisor Comum \\
\hline Máximo Múltiplo Comum & Critérios de Divisibilidade \\
\hline Fenômenos Periódicos & Divisão Euclidiana \\
\hline Frações & Problemas Lógicos \\
\hline
\end{tabular}

A tabela 2 apresenta os sub-temas encontrado e utilizados para classificar as questões extraídas do banco. Esta classificação ocorre em paralelo com a extração das propriedades do problema, ambos sub-fases da seleção. A extração das propriedades consiste em abstrair as variáveis e a lógica do problema. Após isto, mais problemas com o raciocínio semelhante poderiam ser escolhidos e criados.

Um exemplo a ser citado é a questão "O passeio no parque de João", retirado do banco de questões de 2010 (Fase de Seleção do banco e Análise Referencial). O problema escolhido foi modificado baseado nos critérios expostos anteriormente para se 
adequar ao subtema da Divisão Euclidiana. O enunciado explana que João caminha todo dia por um parque, onde o mesmo toca todas as árvores, disponibilizadas em um sentido horário. O discente, tendo ciência destes dados, deve descobrir qual a árvore $\mathrm{N}$ tocada pelo personagem.

Dado o ponto de partida e a direção, espera-se que o discente utilize o método da divisão euclidiana para descobrir a última árvore tocada por João. Este método pode ser descrito como $A=B \times Q+R$, onde $A$ é o número de toques de João e $B$ é o número de árvores. $Q$ é a variável multiplicadora encontrada na divisão e $R$ é o resto, que será utilizado para encontrar a resposta (Fases de Classificação e Extração das propriedades). Após essa fase, pode-se desenvolver o algoritmo para gerar os valores randômicos do problema (Figura 2).

1: Função Questão114(arvore_escolhida)

2: nDeArvores $=$ nAleatorio $(100)+10$

3: posicao $=$ nDeArvores $\% 12$

4: se (arvore_escolhida $==$ posicao)

5: retorne Verdadeiro

6: senão

7: retorne Falso

8: end

Figura 2. Algoritmo da questão 114

Pode-se observar que o número de árvores é uma variável que pode ser alterada, influenciando na resposta e com isso, gerando outra questão a ser inserida no banco. Outro valor que pode ser alterado é a posição inicial e a direção do passeio de João. Estas são algumas propriedades que podem ser alteradas para aumentar a variação da questão.

Por fim, para analisar o processo de Gamification e a aplicação no geral, é utilizado o método proposto por [Whitton, 2009]. Este consiste na análise de um conjunto de critérios e elementos que tornam eficiente a interface e a aplicação baseada em Digitalgame based learning. A tabela 3 apresentam as métricas utilizadas para a medição da eficiência do método no sistema.

Segundo [Whitton, 2009] os doze conceitos apresentados na tabela 3 devem ser considerados como indicadores de eficiência do design educacional. Isto se deve ao fato de que há jogos que não necessariamente cobrem todos os critérios e tem propriedades pedagógicas eficientes.

A implementação do sistema, assim como os problemas, ocorreu por meio da linguagem de programação $\mathrm{PhP}$ em conjunto com o framework CakePhP, uma vez que ambos oferecem todos os recursos necessários para o desenvolvimento da plataforma.

\section{Sistema Desenvolvido}

Baseado no que foi exposto na seção 3, o sistema possui uma divisão de roles, ou categorias, de usuários: Administradores e Discentes. Os administradores tem a permissão de cadastrar novos temas no sistema, assim como sub-temas e novos problemas (Figura 3).

Conforme pode ser visualizado na figura 3, após a autenticação o administrador poderá criar novos temas, subtemas ou problemas, assim como editá-los e visualizá-los. 


\begin{tabular}{|c|c|}
\hline Critério & Exemplos de implementação \\
\hline Auxílio no aprendizado ativo & Encorajar exploração e solução de problemas. \\
\hline Gerar motivação & $\begin{array}{l}\text { Alto nível de interatividade, tarefas com níveis adaptáveis e } \\
\text { controles intuitivos. }\end{array}$ \\
\hline Adequação & $\begin{array}{l}\text { Garantir que o conteúdo esteja de acordo com as atividades } \\
\text { aplicadas. }\end{array}$ \\
\hline Auxílio na reflexão & $\begin{array}{l}\text { Garante um espaço ao discente para que ele pense nos con- } \\
\text { ceitos utilizados para a resolução dos problemas. }\end{array}$ \\
\hline $\begin{array}{l}\text { Fornecer experiência equitati- } \\
\text { vas }\end{array}$ & Prover oportunidades iguais á todos os participantes. \\
\hline Fornecer suporte contínuo & $\begin{array}{l}\text { Prover orientações e supervisões sobre as atividades dos } \\
\text { alunos. }\end{array}$ \\
\hline Critério de interface & Exemplos de implementação \\
\hline Interação flexível & $\begin{array}{l}\text { Tornar claro o propósito das interações do usuário com con- } \\
\text { troles lógicos e consistentes. }\end{array}$ \\
\hline Suporte à comunidade & $\begin{array}{l}\text { Fornecer maneiras eficientes para a comunicação entre os } \\
\text { usuários, além de usar atributos que permitam o usuário se } \\
\text { identificar na comunidade. }\end{array}$ \\
\hline Navegação transparente & $\begin{array}{l}\text { Fornecer meios alternativos de navegação, fornecer uma } \\
\text { visão geral do usuário dentro do sistema. }\end{array}$ \\
\hline Controle de usuários & $\begin{array}{l}\text { Fornecer níveis e problemas auto-ajustáveis, permitir que as } \\
\text { tarefas possam ser realizadas a qualquer momento. }\end{array}$ \\
\hline Robustez & $\begin{array}{l}\text { Facilidade para se recuperar de erros fatais, garantir que a } \\
\text { interface seja responsiva aos comandos do usuário. }\end{array}$ \\
\hline Interface visual adequada & $\begin{array}{l}\text { Desenvolver a interface de modo que seja simples e estetica- } \\
\text { mente prazerosa ao indivíduo, além de utilizar componentes } \\
\text { consistentes. }\end{array}$ \\
\hline
\end{tabular}

O módulo também permite que o administrador tenha uma visão geral sobre as estatísticas relacionadas a cada um dos itens citados.

Já a seção dos Discentes é limitada a edição do perfil, visualização do mesmo, estatísticas e conquistas. As conquistas estão relacionadas as estatísticas do usuário que são armazenadas pelo sistema (Figura 3).

O estudante também pode escolher o nível, o tema e resolver as questões referentes. Após realizar uma série de questões, pode visualizar os resultados e começar novamente. O nível, tema e subtemas dos problemas são cadastrados juntos com os mesmos (Figura 3).

Para o cadastro das questões foram criados campos onde devem ser inseridos o enunciado e a lógica de implementação, na linguagem PhP (Figura 4). No enunciado, o elaborador deve especificar quais as variáveis que poderão ser alteradas quando forem gerados os valores.

O processo de inserção no sistema não é algo trivial, uma vez que é necessário um 


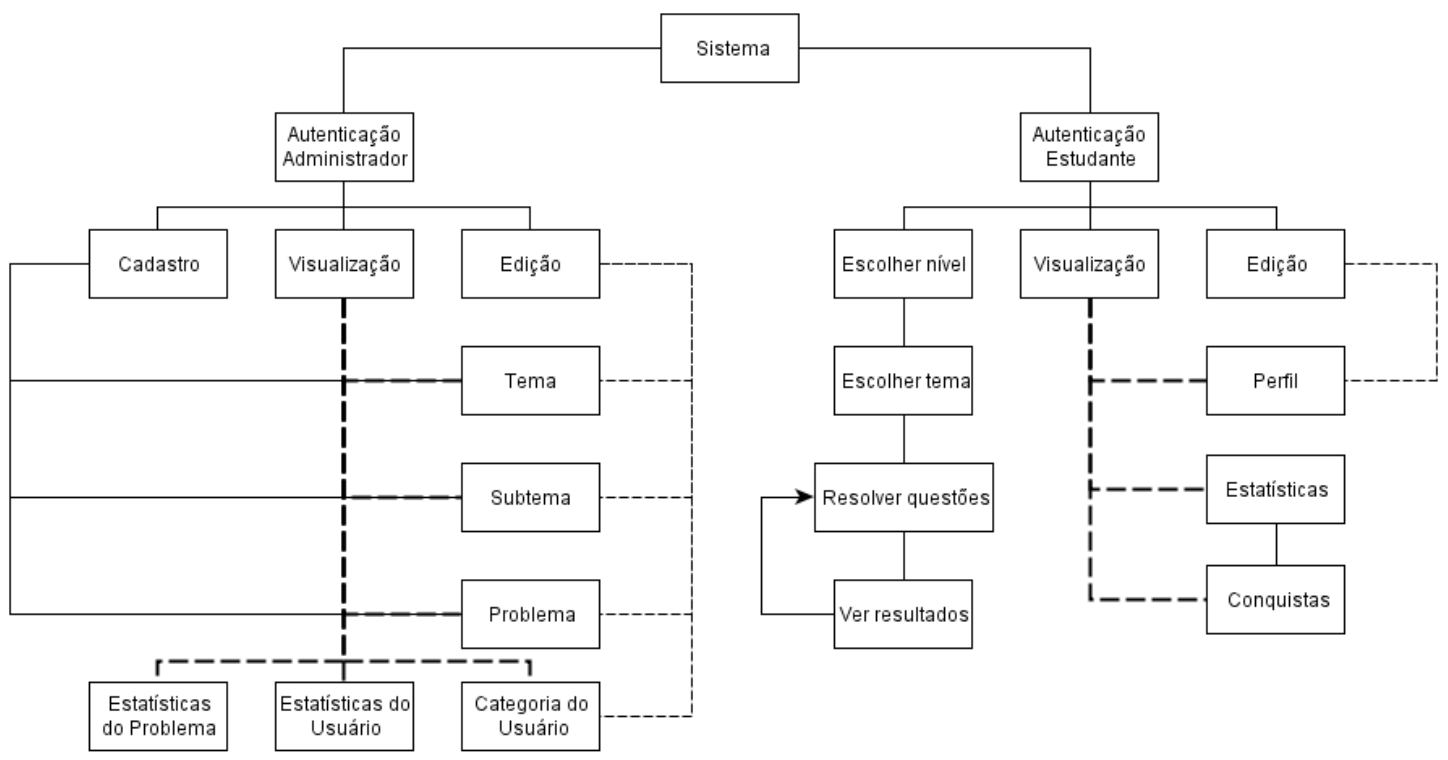

Figura 3. Módulos de funcionalidades do administrador e estudante

treinamento prévio do elaborador da questão, relacionado a linguagem de programação $\mathrm{PhP}$ e as variáveis do problema, para que esta seja implementada no sistema. Para que este possa implementar de maneira correta o algoritmo da questão.

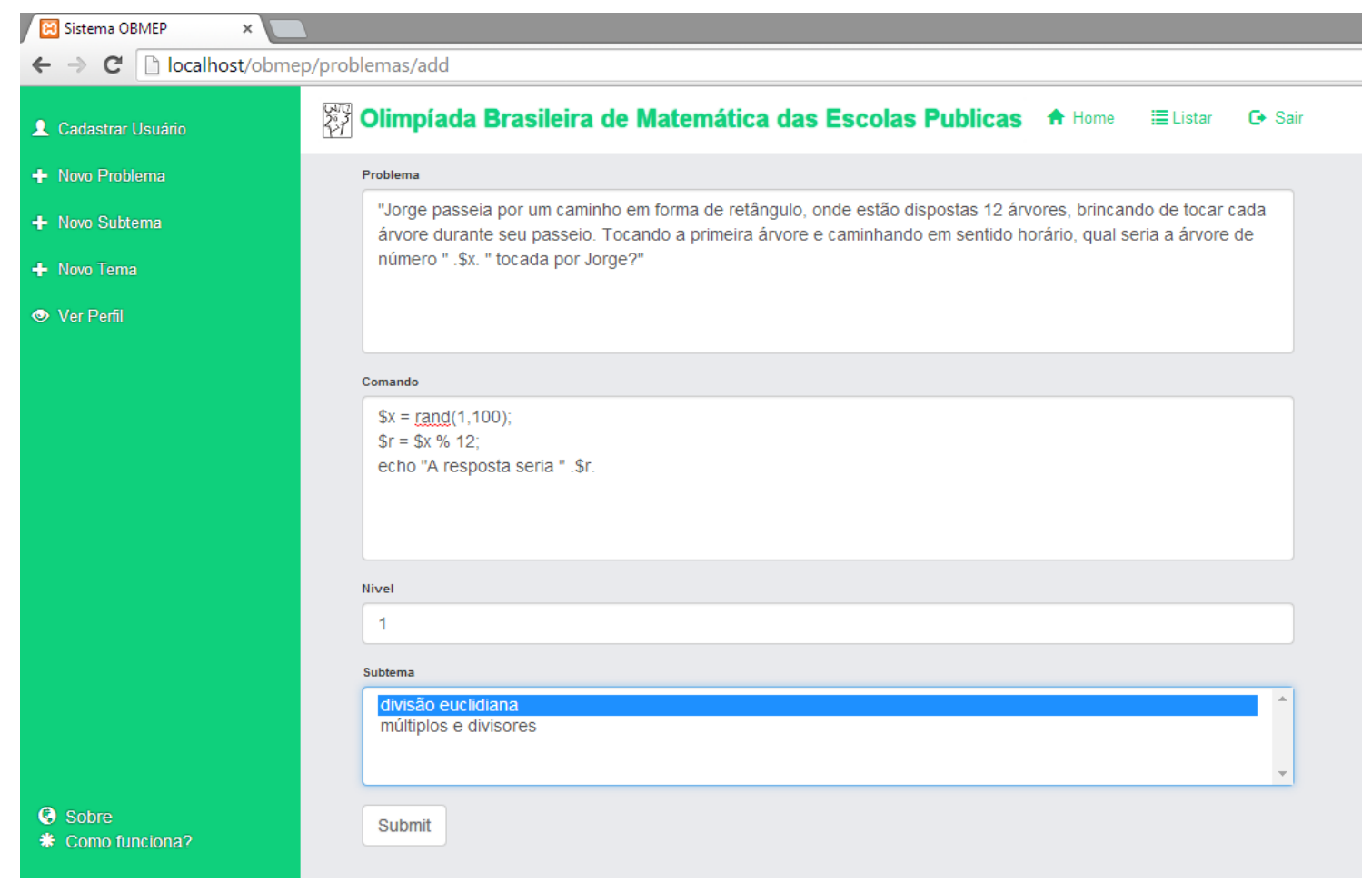

Figura 4. Seção de cadastro das questões

Na figura 4 é apresentada a tela de cadastro de questões. O Problema caracteriza o enunciado da questão, enquanto o Comando determina o trecho de código ou função 
utilizados para gerar os valores dos problemas a serem resolvidos. O nível determina a dificuldade da questão e o subtema, a classificação das mesmas.

Além disto, o usuário também tem acesso as estatísticas geradas pela utilização do sistema. Como a porcentagem de questões respondidas corretamente e incorretamente, o total de questões respondidas e o tempo total de utilização do sistema. Esta última variável é incrementada somente quando o usuário está resolvendo os problemas.

A cada questão resolvida, o sistema recalcula o tempo médio de resolução da questão, com a finalidade de tornar os pontos adaptativos, a medida que mais usuários a resolvem. Estas estatísticas também estão presentes no problema, assim é possível ter uma visão geral das maiores dificuldades encontradas pelos discentes, assim como as sub-áreas influenciadas.

\subsection{Conceitos de Gamification utilizados}

Em relação aos conceitos de Gamification aplicados ao sistema, foram implementados: Objetivos, Competição, Oportunidades, Pressão Temporal, Novidades, Níveis, Renovação, Dados, Progresso, Pontos, Reconhecimento e Classificação. O objetivo principal consiste na resolução do maior número de questões, da maneira correta, para alcançar novos níveis e assim treinar os conhecimentos dos discentes para prepará-los para as próximas olimpíadas.

A competição é visualizada por meio da implementação de placares, com o intuito de criar uma rivalidade saudável entre os discentes. Já as oportunidades são a implementação da aleatoriedade na geração dos problemas. A pressão temporal consiste no tempo como fator para a geração de conquistas durante a resolução das questões. A novidade permite que o usuário tenha acesso a novos problemas, temas e subtemas a medida que ele progride no sistema.

Níveis, pontos e progresso são conceitos interligados, uma vez que o estudante adquire os pontos para subir de nível e isto é demonstrado pelo progresso. O reconhecimento é representado pelas conquistas adquiridas pelo estudante ao desempenhar determinadas tarefas no sistema, como resolver um número específico de questões em um espaço de tempo curto.

Por fim, a mecânica da renovação está presente na opção de refazer os problemas previamente resolvidos, além de reiniciar os valores dos placares periodicamente. As categorias fornecem títulos aos jogadores, juntamente com as conquistas relacionadas a cada um e os dados são visualizados pelo jogador como informações referentes aos problemas que estão sendo resolvidos.

Sobre estes conceitos implementados no sistema, foi realizada a avaliação de acordo com os critérios definidos por [Whitton, 2009]. Com o intuito de verificar se a aplicação está apta à ser aplicada em discentes, uma vez que esta já foi aprovada por docentes envolvidos no projeto.

\section{Discussão}

Baseando-se nos critérios expostos, é válido afirmar que a aplicação está de acordo com os critérios: Auxílio no aprendizado ativo, geração de motivação, adequação, auxílio na reflexão, fornecimento de experiências equitativas e o suporte contínuo. Já em relação 
aos critérios de interface: possui interação flexível, suporte à comunidade, navegação transparente, controle de usuários e robustez.

O auxílio no aprendizado ativo está presente no encorajamento na resolução de problemas pelo discente, assim como garantir os objetivos do jogo e alinhá-los aos objetivos pedagógicos esperados. A geração de motivação se dá por meio dos objetivos claros e realizáveis, além de fornecer controle sobre as propriedades de ensino implementadas (usuário pode visualizar dicas, por meio dos conceitos dos assuntos que estão no sistema).

A adequação está presente nos problemas estarem diretamente ligados aos conceitos vistos pelos discentes. Já o auxílio na reflexão pode ser visualizado no foco em ensinar e treinar os conceitos matemáticos do discente. As experiências equitativas estão presentes em apresentar o conteúdo referente ao nível dos estudantes e possibilitar que um resolva os mesmos problemas que os demais.

Por fim, o suporte contínuo está presente nas respostas do sistema em relação as ações do usuário. Ao responder corretamente um problema, a aplicação fornece instantaneamente ofeedback do aluno. Estas informações também são armazenadas com o intuito de melhorar o sistema e os problemas implementados.

Já em relação a interface, de acordo com os docentes, possui: interação flexível, uma vez que o propósito das interações é intuitivo e consistente. Também possui o suporte à comunidade uma vez que os usuários podem relatar qualquer mal funcionamento com a aplicação diretamente com os administradores, além de se comunicarem de maneira objetiva.

A navegação transparente é visualizada no momento em que o usuário realiza o login no sistema, e pode navegar e retornar entre as diversas seções a partir do menu de usuário. Este pode ser visualizado no lado esquerdo da aplicação, na figura 4. O controle de usuários é visualizado pelo conceito da renovação em conjunto com o de oportunidade.

A robustez do sistema é garantida pela utilização do framework CakePhP, além da interface ter um tempo de resposta apto para as ações realizadas pelo usuário. Por fim o sistema possui uma interface visual adequada, apontando as diversas opções e comandos que podem ser realizados pelo usuário, sem a necessidade de uma intervenção do sistema.

\section{Conclusões e Trabalhos Futuros}

A partir do estudos realizados, foi proposto o desenvolvimento de um sistema web para auxiliar no estudo e treinamento, para as Olimpíadas Brasileiras de Matemática, de discentes do Ensino Fundamental. Também como proposta fora implementados os conceitos de Gamification com a finalidade de tornar a experiência mais prazerosa e engajante para o usuário.

O sistema encontra-se em fase alpha e já é possível cadastrar usuários (assim como atribuir as roles), temas, sub-temas, questões e os conceitos de Gamification. A aplicação também foi avaliada por docentes da área de matemática com o intuito de verificar os critérios descritos na pesquisa de [Whitton, 2009]. Esta validação atesta que a aplicação já pode ser aplicada com discentes para avaliar o desempenho dos mesmos.

Como trabalhos futuros estão previstos a utilização da aplicação por um grupo de discentes, de escolas públicas, do quinto e sexto ano e que pretendem participar das 
próximas olimpíadas de matemática. Assim como alunos que não tenham tanta afinidade com a área com o intuito de motivá-los a estudar mais sobre a mesma.

Outro trabalho previsto é a utilização das estatísticas do sistema para verificar o desempenho dos usuários em determinadas áreas. Com isso é possível mapear os subtemas que os discentes tem mais dificuldade. Juntamente com as estatísticas geradas pelas questões, será possível realizar uma análise mais profunda sobre as propriedades de cada questão e cada sub-tema.

\section{Referências}

Olimpíada Brasileira de Matemática das Escolas Públicas - OBMEP.

\section{PROGRAMME FOR INTERNATIONAL STUDENTS ASSESSMENT (PISA) RE-} SULTS FROM PISA 2012.

Csikszentmihalyi, M. (2008). Flow: The Psychology of Optimal Experience.

Deterding, S., Dixon, D., Khaled, R., and Nacke, L. (2011). From game design elements to gamefulness: defining gamification. Proceedings of the 15th ..., pages 9-15.

Dignan, A. (2011). Game Frame. Free Press.

Generoso, A. A. P., Neto, J. a. C., Reinehr, S., and Malucelli, A. (2013). Abordagem Qualitativa do uso das TDIC na Educação Básica. Anais do XIX Workshop de Informática na Escola (WIE 2013), (Cbie):230-239.

Kapp, K. M. (2012). The Gamification of Learning and Instruction: Game-based Methods and Strategies for Training and Education.

Lee, J. and Hammer, J. (2011). Gamification in Education: What, How, Why Bother? Academic Exchange Quarterly, 15:1-5.

McGonigal, J. (2011). Reality is broken: Why games make us better and how they can change the world. Number Deterding. Penguin Group, The.

Mesquita, M., Toda, A., Brancher, J., and do Carmo, R. (2013). Utilizing Gamification concepts tied with Social Networks to support students in programming classes. Proceedings of the XV Simpósio Internacional de Informática Educativa, pages 127-132.

Monteiro, M., Ferneda, E., Takaki, W., Gomes, R., Junior, F., and Costa, E. (2006). Um Ambiente Lúdico de Ensino e Aprendizagem de Matemática Fundamental em Plataforma Web. Anais do Simpósio Brasileiro de Informática Educativa, pages 67-69.

Nelson, M. (2012). Soviet and American precursors to the gamification of work. Proceeding of the 16th International Academic ..., pages 23-26.

Pietruchinski, M., Neto, J. C., Malucelli, A., and Reinehr, S. (2011). Os jogos educativos no contexto do SBIE: uma revisão sistemática de Literatura. Anais do Simpósio Brasileiro de Informática Educativa, pages 476-485.

Salen, K. and Zimmerman, E. (2003). Rules of Play: Game Design Fundamentals.

Whitton, N. (2009). Games-Based Learning Advancements for Multi-Sensory Human Computer Interfaces. IGI Global.

Zichermann, G. and Cunningham, C. (2011). Gamification by Design: Implementing Game Mechanics in Web and Mobile Apps [Paperback]. O'Reilly Media; 1 edition. 